\title{
Magnetic Resonance and Computed Tomography Imaging of Arrhythmogenic Right Ventricular Dysplasia
}

\author{
Harikrishna Tandri, MD, ${ }^{1}$ Chandra Bomma, MD, ${ }^{1}$ Hugh Calkins, MD, ${ }^{1}$ and \\ David A. Bluemke, MD, $\mathrm{PhD}^{2 *}$
}

Arrhythmogenic right ventricular dysplasia (ARVD) is a familial cardiomyopathy that causes fibro-fatty replacement of the right ventricle (RV), leading to sudden death due to ventricular arrhythmias. The disease is an important cause of sudden death in individuals younger than 35 years of age. Structural and functional abnormalities of the RV constitute an important diagnostic criterion for the disease. Diagnosis of ARVD is often a challenge as conventional imaging modalities have significant limitations to visualize the RV. Recently, magnetic resonance imaging (MRI) and computed tomographic (CT) imaging have emerged as robust clinical tools for evaluation of myocardial pathology. In addition to providing morphologic and functional information, both imaging modalities have the ability to demonstrate intramyocardial fat, which is the pathological hallmark in ARVD. This article discusses the current status and role of MRI and CT imaging in the diagnosis of ARVD.

Key Words: MRI; CT; right ventricular dysplasia; cardiomyopathy; diagnosis

J. Magn. Reson. Imaging 2004; 19:848-858.

(C) 2004 Wiley-Liss, Inc.

ARRHYTHMOGENIC RIGHT VENTRICULAR dysplasia (ARVD) is a genetic cardiomyopathy characterized by fibro-fatty replacement of the right ventricular (RV) musculature, which leads to ventricular arrhythmias and RV failure $(1,2)$. This condition accounts for up to $5 \%$ of sudden deaths in young individuals less than 35 years of age in the United States, and $25 \%$ of exerciserelated deaths in the Veneto region of Italy $(3,4)$. The

${ }^{1}$ Division of Cardiology, The Johns Hopkins University, Baltimore, Maryland.

${ }^{2}$ Department of Radiology, The Johns Hopkins University, Baltimore, Maryland.

Contract grant sponsor: National Institutes of Health; Contract grant number: Research Grant 1 UO1 HL65594-01A1; Contract grant sponsor: Johns Hopkins ARVD center (funded by a private grant from the Bogle Foundation)

*Address reprint requests to: D.A.B., MRI, Room 143 (Nelson Basement), The Johns Hopkins Hospital, 600 N. Wolfe St., Baltimore MD 21287. E-mail: dbluemke@jhmi.edu; ;www.arvd.com

Received July 14, 2003; Accepted March 4, 2004

DOI $10.1002 /$ jmri.20078

Published online in Wiley InterScience (www.interscience.wiley.com). exact prevalence of this condition is not known, but is estimated to be around 1 in 5000. The disease rarely manifests before adolescence and usually presents in the second or third decade of life (1). Affected individuals are often men who have an athletic lifestyle. Presenting symptoms vary from asymptomatic individuals with occasional palpitations to syncope or even to sudden death. The disease is inherited in an autosomal dominant fashion. A common clinical scenario is the death of a family member with autopsy showing ARVD, which prompts screening of the first-degree relatives.

Diagnosis of ARVD presents a challenge for the clinician, as the nature of RV involvement in early stages of ARVD is poorly understood. The diagnosis is based on a set of major and minor criteria proposed by the Task Force of cardiomyopathies in 1994 (5). These criteria encompass electrical, anatomic, and functional abnormalities that are a consequence of progressive fibrofatty infiltration, which results in loss of RV myocytes. Structurally, this manifests as regional reduction in wall thickness, wall hypertrophy, and focal bulging of the RV wall in diastole. Focal or global contraction abnormalities, chamber enlargement, enlarged RV outflow tract (RVOT), and RV aneurysms have been described. Accurate and detailed evaluation of the RV structure and function is essential for the diagnosis and for screening of the first-degree relatives.

Noninvasive modalities commonly used to evaluate the RV include two-dimensional echocardiography and radionuclide ventriculography. Both modalities have significant limitations in assessing the RV due to its complex geometry $(6,7)$. Because RV involvement in ARVD is nonuniform, complete and multiplanar assessment of the RV is desirable. Recently, magnetic resonance imaging (MRI) and computed tomography (CT) have emerged as robust tools to evaluate cardiac structure and function (8-11). Both techniques can provide direct evidence of fatty infiltration and structural alterations of the $\operatorname{RV}(12,13)$. The noninvasive nature of these investigations, multiplane capability, and unique ability to provide tissue characterization are ideal for assessment of ARVD. The purpose of the current review is to discuss the current status, 
strengths, and limitations of MRI and cardiac CT in evaluation of patients with suspected ARVD.

\section{MRI OF ARVD}

Among the current cardiac MR applications in cardiomyopathies, the greatest potential as well as biggest challenges are in the diagnosis of ARVD. Compared to conventional imaging modalities, MR is uniquely suited to evaluate ARVD. The three-dimensional depiction of anatomy by MRI allows both qualitative and quantitative analysis of RV function (14). MR has the ability to demonstrate intramyocardial fat, which is the pathological hallmark of ARVD (12). The last 10 years have seen significant improvements in MR hardware, with tremendous increases in acquisition speed and image quality. The development of ECG gating and the use of breath-hold imaging have significantly reduced motion artifacts. Improved tissue contrast is currently achieved by the use of inversion recovery black-blood imaging techniques for depiction of cardiac morphology (15). Improvements in cine imaging using ECG-gated steady-state free precession imaging (SSFP) pulse sequence have resulted in better delineation of endocardial borders, enabling accurate and reproducible volumetric measurements (14). For these reasons, MRI has been increasingly used in evaluation of the RV and has evolved as the noninvasive modality of choice in ARVD.

Casolo et al (12) were the first to describe the use of MRI to assess ARVD in 1987. They demonstrated intramyocardial fat deposits in the RV on conventional spin echo (SE) imaging in a patient with advanced ARVD. Since that time, several authors, including our group, have reported MR abnormalities in ARVD (1625). Broadly, MRI abnormalities in ARVD can be grouped into two major categories: 1) morphological abnormalities and 2) functional abnormalities. Morphologic abnormalities include intramyocardial fat deposits, focal wall thinning, wall hypertrophy, trabecular disarray, and RVOT enlargement. Functional abnormalities include regional contraction abnormalities, aneurysms, RV global dilation/dysfunction, and RV diastolic dysfunction. The sites of involvement of these abnormalities are observed in the "triangle of dysplasia," which is constituted by the inferior subtricuspid area, RV apex, and RV infundibulum (26) (Fig. 1). The goal of MRI in ARVD is to accurately assess the RV for the presence or absence of these abnormalities, which aids not only in the diagnosis but also in follow-up of patients.

\section{MR Assessment of Cardiac Morphology in ARVD}

Accurate depiction of morphology is very important in most cardiac applications, and ARVD exemplifies this statement. Morphologic evaluation is generally performed by the use of black-blood techniques. Conventional SE pulse sequences were initially used to evaluate cardiac morphology, and the majority of studies in literature have used SE imaging for ARVD. Black blood in the cardiac chambers is obtained by the saturation of inflowing blood signal both above and below the acquired volume. Disadvantages include long acquisition

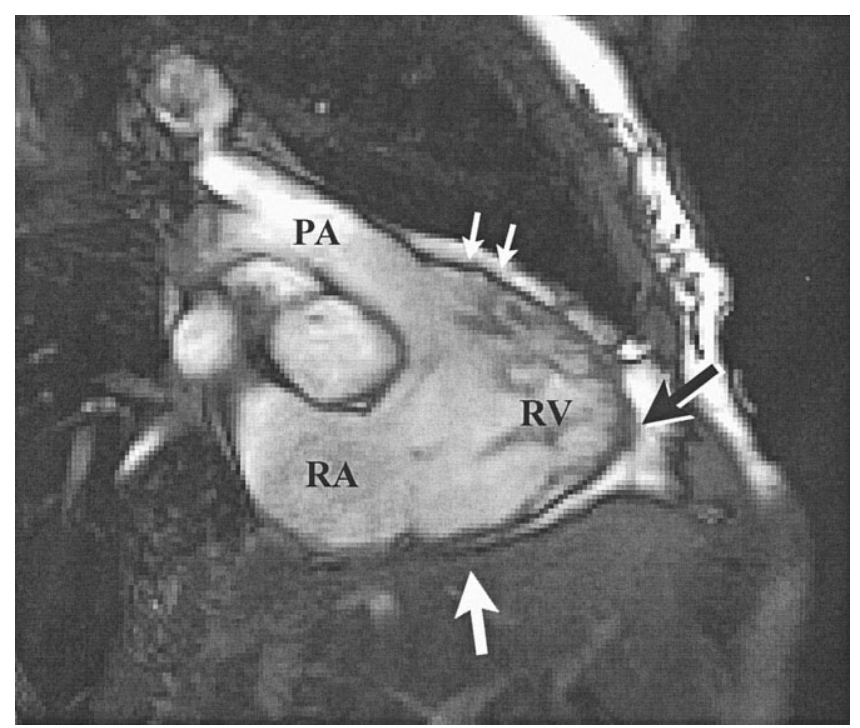

Figure 1. GRE image in the oblique saggital plane showing the triangle of dysplasia, which is constituted by the inferior subtricuspid area (thick white arrow), RV apex (black arrow), and $\mathrm{RV}$ infundibulum (thin white arrows). RA, right atrium; PA, pulmonary artery.

times, precluding breath holding, and resulting motion artifacts, which degrades image quality. Blood suppression is often poor in the long-axis planes and is ineffective for slow flow in the atria and RV.

Currently, black-blood techniques using breath-hold imaging with double-inversion recovery fast SE (DIRFSE) techniques are preferred to traditional SE imaging. Breathhold FSE sequences consistently provide end-diastolic images with minimal motion artifacts and improve resolution of myocardial detail (27-29). Blackblood inversion-prepared half-Fourier single-shot turbo SE (HASTE) imaging has not been systematically evaluated, but is currently not recommended due to blurring of detail with this sequence. A dedicated cardiac coil is recommended for best results, although we use only the anterior coil elements to prevent wraparound artifact when using a small field of view (FOV). An anterior saturation band (Fig. 2) is placed over the anterior subcutaneous fat for further suppression of motion artifacts.

\section{Morphologic Features of ARVD}

\section{Intramyocardial Fat}

Normal myocardium shows an intermediate signal similar to that of skeletal muscle, and fat appears as a hyperintense signal (bright) on black-blood images. Figure 3A shows a black-blood image from a normal volunteer. In normal individuals epicardial fat overlies the $\mathrm{RV}$ and is abundant toward the RV apex and in the atrioventricular groove. There is often a clear line of demarcation between the gray RV myocardium and the epicardial fat. Disruption of this line of demarcation (Fig. 3B) and extension of the hyperintense signals into the RV myocardium are frequently noted in ARVD, indicating infiltration of epicardial fat into the RV wall. 
The prevalence of intramyocardial hyperintense signals in ARVD on T1-weighted SE imaging has ranged from $22 \%$ to $100 \%$ in different studies $(16-25)$. The largest series is by Auffermann et al (20), who imaged 36 biopsy-proven ARVD patients and found intramyocardial hyperintense signals in only $22 \%$ of patients. The patients in this study had different stages of ARVD, and a significant number had localized ARVD. An interesting finding of this study was that fat infiltration on MRI, not on biopsy, predicted inducibility of ventricular tachycardia at electrophysiologic testing. Wichter (21) added 16 additional patients to the series by Auffermann et al (52 total patients) and concluded that patients with extensive ARVD had higher incidence of fatty replacement of the RV, compared to localized forms (96\% vs. 58\%). Menghetti et al (25) described SE MRI findings in 15 ARVD patients diagnosed using the Task Force criteria and reported intramyocardial hyperintense signals in $62 \%$ of patients. The differences in incidence of fat signal in ARVD are largely based on differences in patient selection and the definition of abnormal intramyocardial hyperintense signals.

We used the breath-hold DIR-FSE technique to evaluate intramyocardial fat in ARVD and found a high intramyocardial T1 signal (fat) in 9 of 12 patients $(75 \%)$ who were prospectively diagnosed using the current Task Force criteria (16). The use of spectrally selective fat suppression with the DIR-FSE sequence provided additional evidence of fat infiltration due to high contrast between epicardial fat and the RV myocardium. A fat-suppressed sequence may demonstrate a signal void within the myocardium, in a location where a high signal was observed in the non-fat-suppressed image. This increases the confidence for the diagnosis of fat signal, in particular in exams where the results of nonfat-suppressed images are inconclusive (Fig. 4A and B). Fatty infiltration was more commonly noted in the basal regions (RV inflow and outflow) and less frequently at the RV apex (one of nine patients). Biopsy was abnormal in six of the nine patients and the rest

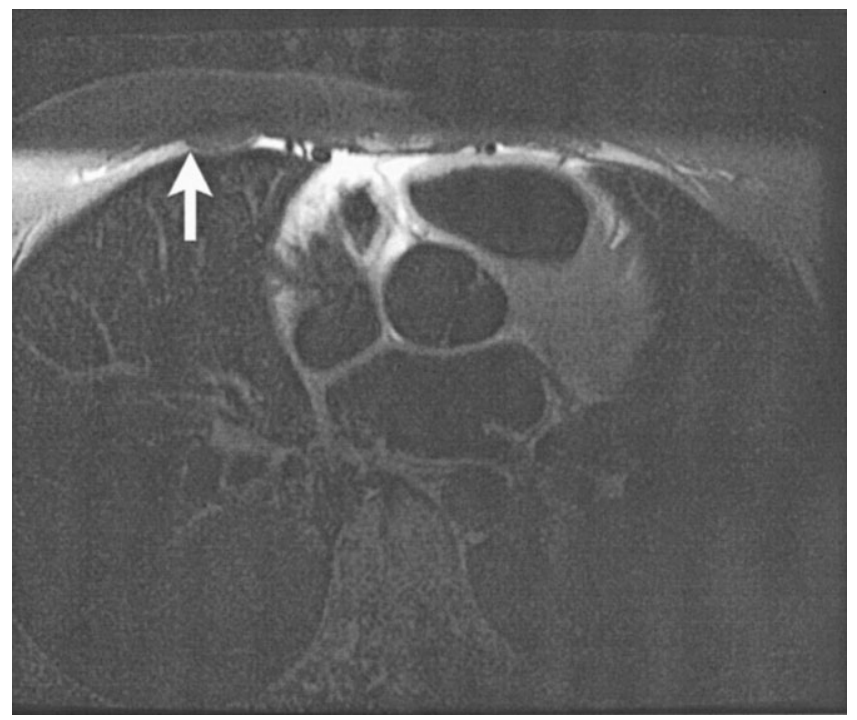

Figure 2. Axial black-blood image showing the location of anterior saturation band (arrow).
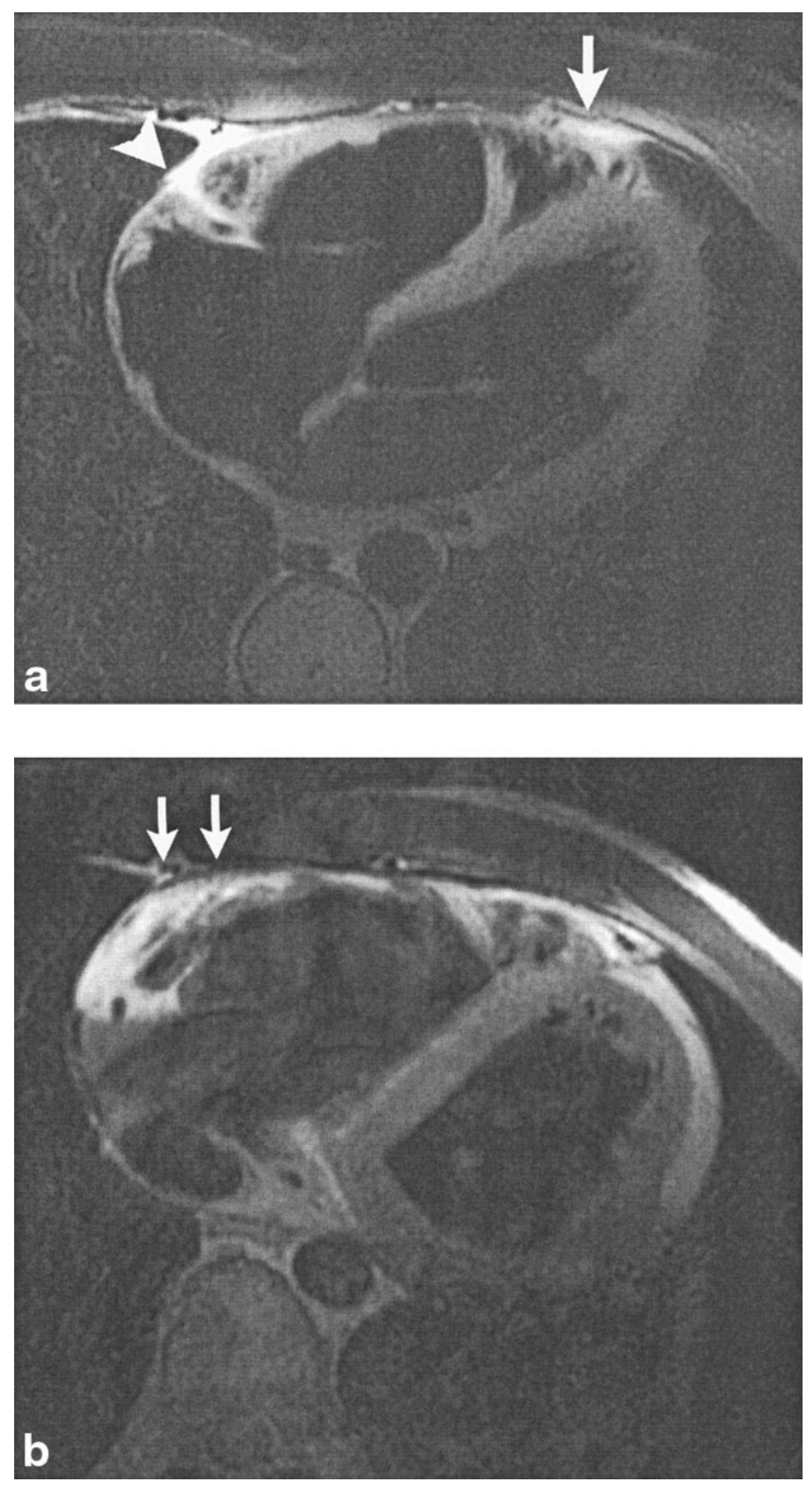

Figure 3. A: Axial black-blood image from a normal volunteer showing a clear line of demarcation between the epicardial fat and the underlying myocardium. Also note the abundance of epicardial fat in the atrioventricular groove (arrowhead) and at the apex (arrow). B: Axial black-blood image from a patient with RV dysplasia showing lack of demarcation between epicardial fat and myocardium (arrows).

had normal biopsies. Biopsies, however, may be inconclusive due to sampling error, since the biopsy site is often the RV septum, which may not be involved in the disease process. Biopsy of the thin anterior RV wall carries a risk of perforation and hemopericardium.

\section{Wall Thinning}

RV wall thinning is defined focal abrupt reduction in wall thickness of $<2 \mathrm{~mm}$, surrounded by regions of normal wall thickness. The exact pathogenesis of wall thinning is not known, but it is thought to be due to apoptotic loss of RV myocytes. Progressive loss of epi- 

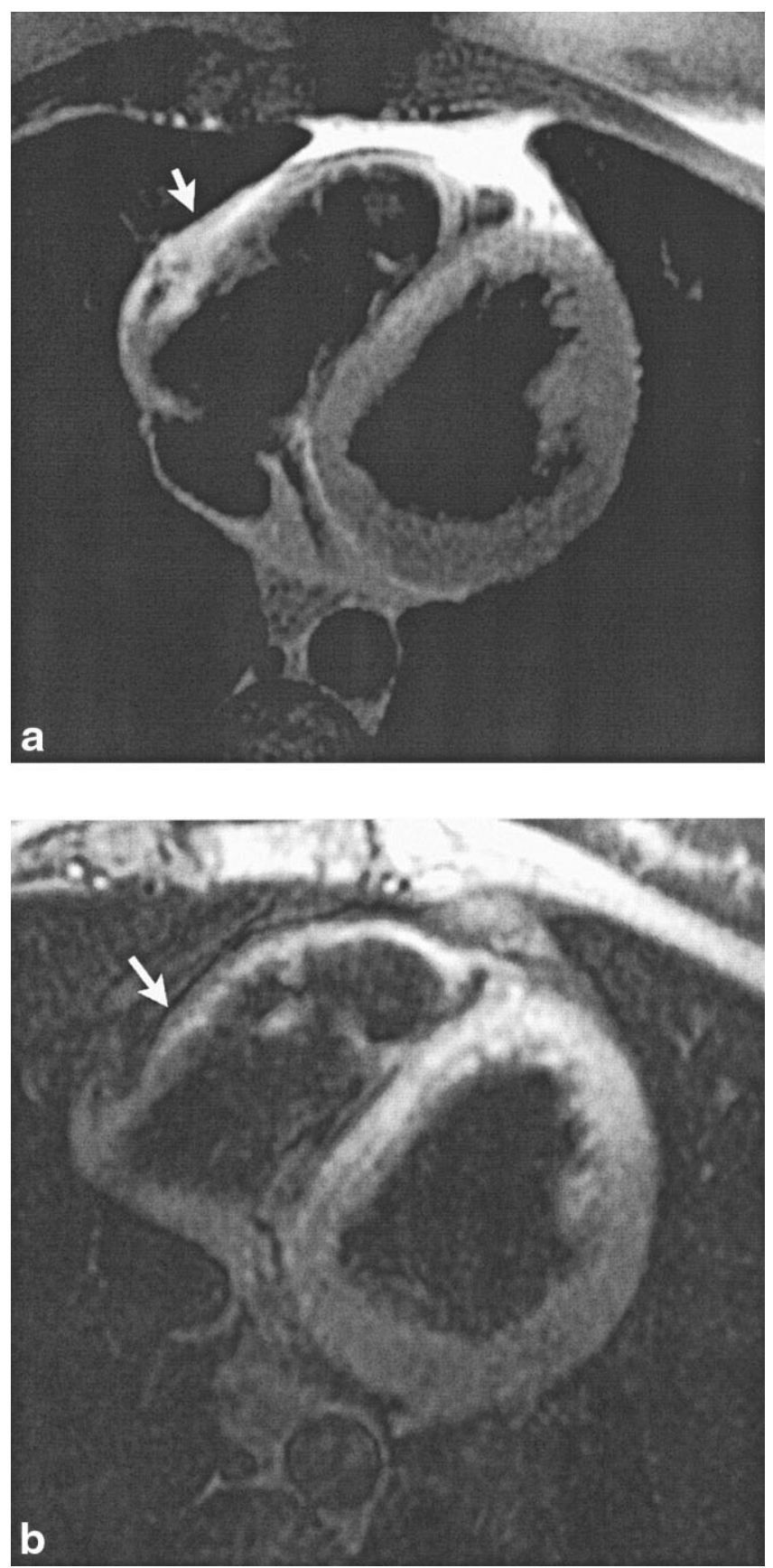

Figure 4. A: Axial black-blood image from a control subject showing heterogeneously increased T1 signal (arrow) toward the RV inflow region. B: Fat-suppressed version at the same level reveals normal RV wall without any abnormality (arrow).

cardial and myocardial layers leaves behind a thin rim of endocardial cells, which are usually not involved until late in the disease process. This finding often observed in pathologic specimens was not revealed in vivo until the emergence of MRI. Compared to intramyocardial fat, fewer reports have addressed the issue of wall thinning in ARVD. Typically the in-plane resolution of DIR-FSE is approximately $1.5 \mathrm{~mm}$. The resolution is further reduced by motion artifacts. Auffermann et al (20) reported wall thinning in 67\% of biopsyproven ARVD patients on SE MRI. In our series we found wall thinning in less than $25 \%$ of patients who met the Task Force criteria (Fig. 5). Wall thickness is often difficult to assess due to adjacent high epicardial fat signal and motion artifacts that are increased in patients with arrhythmias. The use of chemical shift fat suppression may reveal the true thickness of the RV wall, making its identification more reliable.

\section{Wall Hypertrophy}

Wall hypertrophy is defined as RV wall thickness of $>8$ $\mathrm{mm}$. This finding is seldom observed in pathologic specimens as the true RV myocardium is measured exclusive of the epicardial fat (30). In vivo this differentiation is sometimes not possible due to extensive fibro-fatty infiltration with loss of distinction between epicardial fat and the true myocardium. In such cases the RV wall appears hypertrophied with MR images showing islands of gray muscle surrounded by bright signals compatible with fat. This finding was observed in 5 of the 12 patients $(42 \%)$ in our series. Use of fat suppression reveals multiple signal voids within the RV myocardium in locations that showed hyperintense signals in the non-fat-suppressed images (Fig. 6A and B).

\section{Trabecular Disarray}

Molinari et al (24) were the first to describe giant Yshaped trabeculae and hypertrophy of the moderator band in patients with ARVD. This finding has been equated to the angiographic finding of deep fissures with a "pile d'assiettes" (stack of plates) appearance. We found a prevalence (40\%) of trabecular hypertrophy and disarray similar to that of the above study in ARVD patients (Fig. 7). This finding is not specific for ARVD

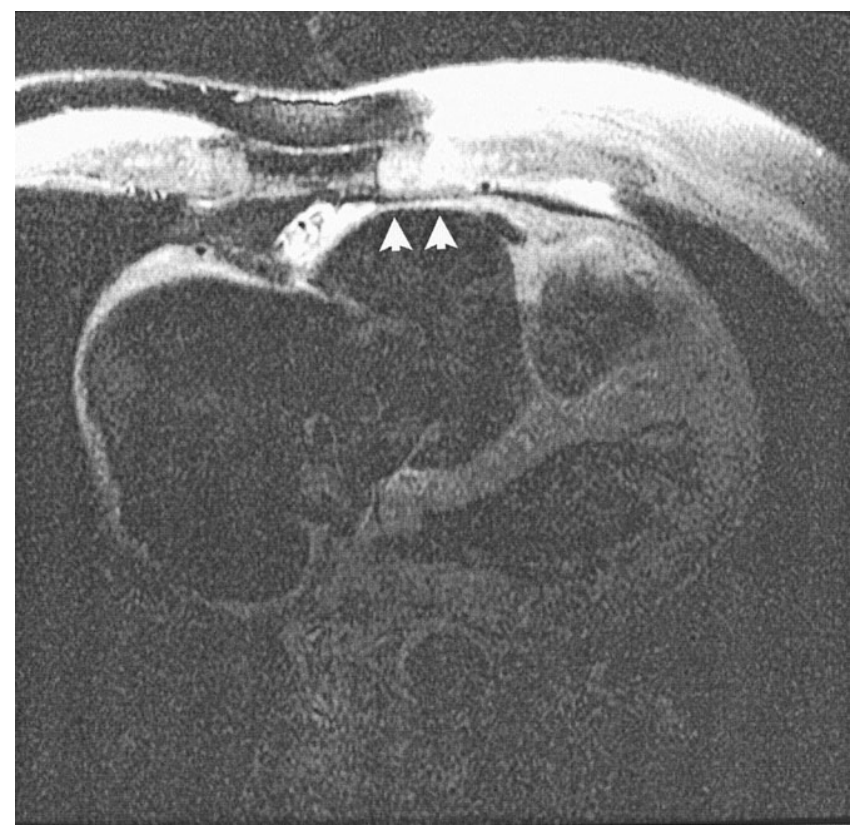

Figure 5. Fat-suppressed axial black-blood image from a patient with RV dysplasia showing focal abrupt thinning (arrowheads) of the anterior wall of the RV. 

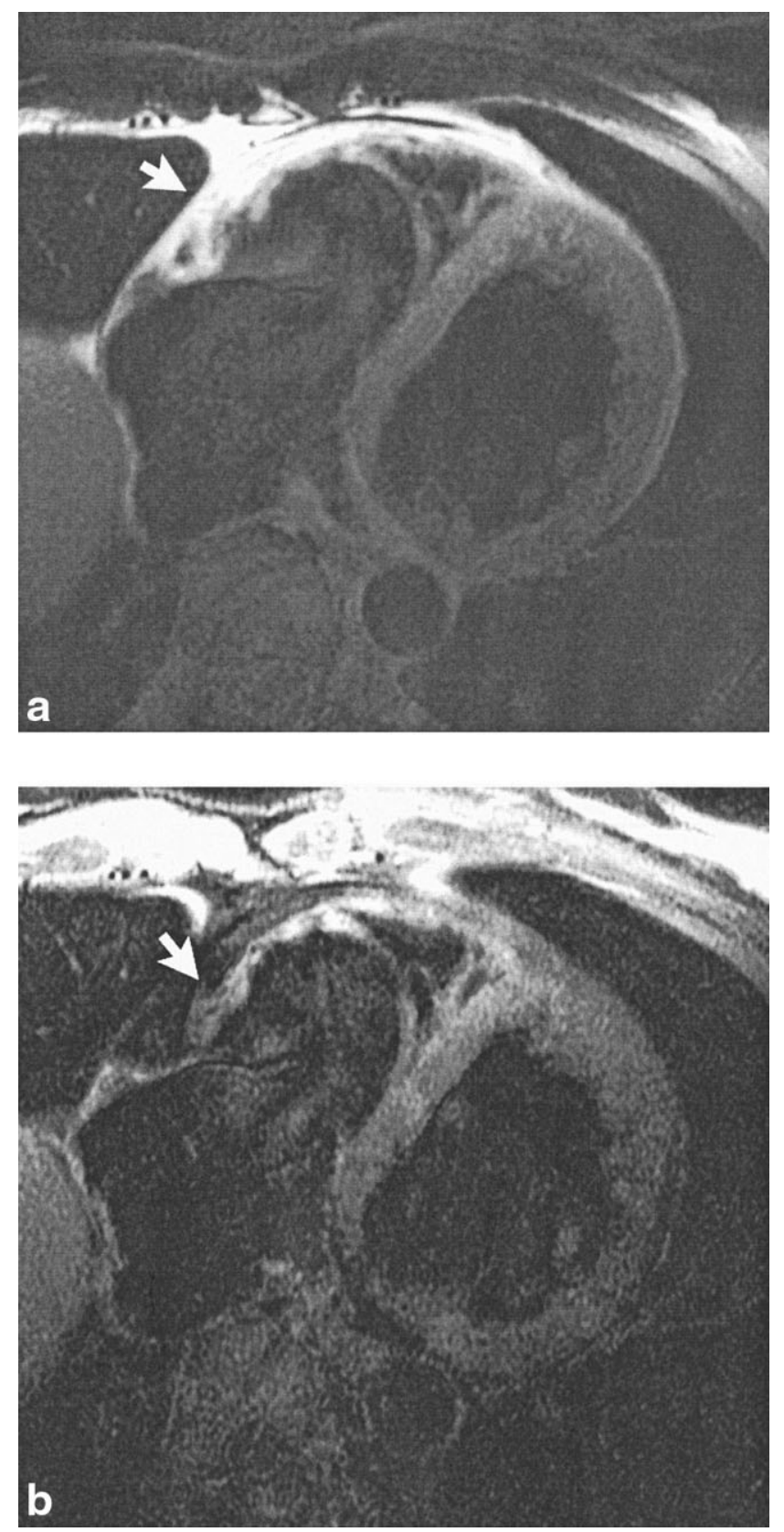

Figure 6. A: Axial black-blood image from a patient with RV dysplasia showing heterogeneously increased T1 signal in the RV anterior wall (arrow). B: Fat-suppressed image at the same level shows multiple signal voids in the same location of the hyperintense signals on the non-fat-suppressed image (arrow).

and may be present in any condition that results in RV hypertrophy or enlargement.

\section{RVOT Enlargement}

The RVOT is a common location for localized ARVD. The $\mathrm{RV}$ outflow is usually equal to or marginally smaller than the aortic outflow tract at the level of the aortic valve. An exception to this rule is pediatric patients in whom the RVOT may be larger than the left ventricular outflow. Presence of an enlarged RVOT beyond adolescence is uncommon. Ricci et al (22) reported enlarged

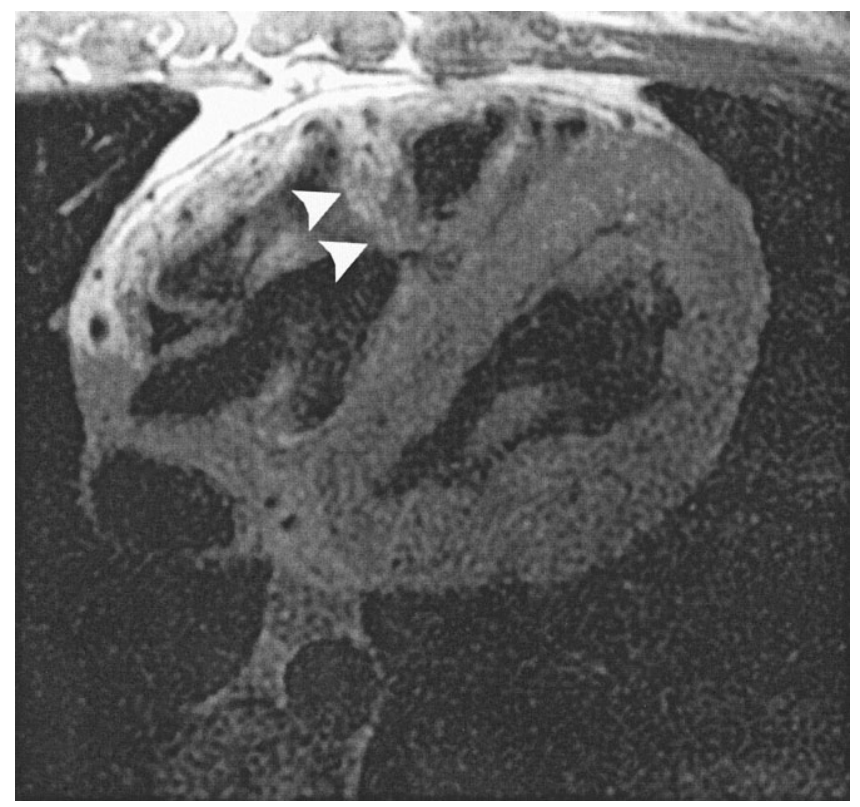

Figure 7. Axial black-blood image from a patient with RV dysplasia showing hypertrophied trabeculations (arrowheads).

RVOT in 15 patients with ARVD, compared to patients with dilated cardiomyopathy. In this study, ARVD patients also had increased RV end-diastolic diameters and volumes compatible with RV dilation. This suggests that RVOT enlargement may be a part of generalized dilation involving the right heart. More important than a simple enlargement is a dysmorphic appearance of the outflow tract (Fig. 8). Abnormal appearance of the RVOT, which is dyskinetic in systole, is highly suggestive of ARVD in the absence of pulmonary hypertension.

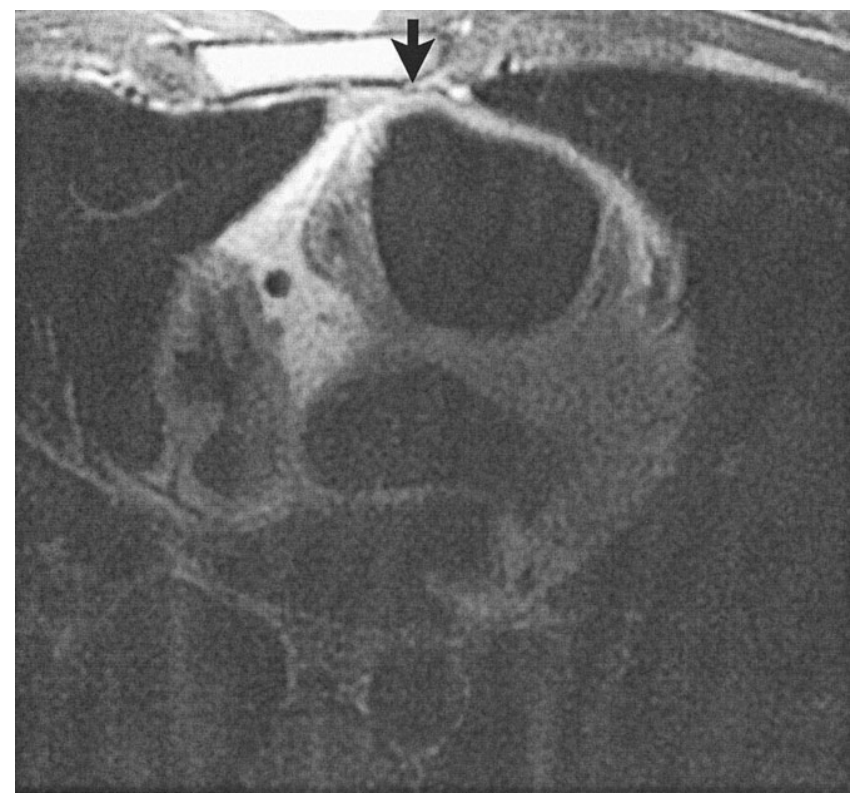

Figure 8. Axial black-blood image from a patient with RV dysplasia showing enlarged and dysmorphic outflow tract with focal bulging anteriorly (arrow). 


\section{MR Assessment of Cardiac Function in ARVD}

One of the major advances in MR cardiac imaging has been the ability to accurately assess global and regional ventricular function without using geometric assumptions. Until the availability of cine MRI, the complex geometry of the RV made quantification by conventional two-dimensional techniques impossible. The techniques to image cardiac function are called brightblood techniques, derived from the appearance of intracavitary blood. These techniques yield both morphologic and functional data. Multiple consecutive images that are acquired with a high temporal resolution can be viewed dynamically to generate functional information. Ventricular volumes and masses using brightblood imaging have been shown to be accurate and reproducible, and MRI is considered the standard of reference $(31,32)$. Although a number of sequences exist for bright-blood imaging, SSFP imaging is the most preferred technique (FIESTA, true FISP, Balanced Fast Field Echo). SSFP sequences result in improved contrast between the blood pool and the myocardium with better delineation of papillary muscles and trabeculation compared to segmented $k$-space cine gradient echo (GRE) images (33). Blood signal is hyperintense on SSFP images, and this is especially important in imaging the RV. If SSFP is not available, segmented $k$-space cine gradient echo images (e.g., fast low-angle shot (FLASH), fast cardiac-gated gradient echo (FASTCARD)) can be used. Conventional cine $k$-space GRE images rely on flowing blood to generate bright blood. In the dysfunctional RV, blood velocities are reduced and signal intensity decreases with conventional GRE imaging. With SSFP, the signal intensity remains high since the signal intensity is proportional to $\mathrm{T} 2$ time.

Global functional assessment is useful and can be accurately performed using cine imaging. But it is well known that ejection fraction reduction and ventricular dilation may not be sensitive indicators of myocardial pathology. This is especially true of ARVD where regional functional abnormalities often precede changes in global function. The multiplane nature of MRI, the high contrast between the blood pool and myocardium, and the high spatial resolution have made it possible to visually assess regional RV dysfunction, which has been the focus of several authors in ARVD assessment. There exists an excellent correlation between MRI and RV angiography (17), the later being the traditional gold standard for RV function. For the above reasons, MRI is a noninvasive alternative for RV functional assessment in screening of first-degree relatives for ARVD and also for follow-up.

\section{Functional Abnormalities in ARVD}

\section{Global RV Dilation/Dysfunction}

Fibro-fatty replacement of the RV in ARVD eventually leads to RV dilation and dysfunction. RV dysfunction is often asymptomatic (34), but is preceded by symptoms related to an associated arrhythmia. MRI does not offer any advantage in patients who have severe reduction in RV function (ejection fraction $<35 \%$ ), as this can be detected even on echocardiography. The advantage of
MRI is to be able to detect minor changes in ventricular volumes over time, which provides insight into an evolving disease process. MR has the capability to accurately and quantitatively measure ventricular volumes, and minor increases in RV end-diastolic volumes may be a subtle and early sign of ARVD. Auffermann et al (20) reported an increased RV end-diastolic volume index in 10 patients with ARVD who were inducible on electrophysiologic studies, compared to control subjects. The $\mathrm{RV}$ volume indices and the global function of ARVD patients, who were noninducible, did not differ from those of the control subjects, suggesting that the patients in this group had localized ARVD. Several other authors have reported RV enlargement and dysfunction using a variety of patient selection criteria. In our report we found that a majority of patients (75\%) who meet the Task Force criteria have some degree of RV enlargement and dysfunction at presentation. Also, there was a linear correlation between the RV end-diastolic volumes and the duration of symptoms, suggesting the progressive nature of the disease (16). Serial quantitative volumetric assessment of RV may play an important role in assessing disease progression and may have an important role in evaluation of first-degree relatives.

\section{Regional Dysfunction}

Much remains to be understood about the pathophysiology of regional dysfunction in ARVD, but regional dysfunction is generally thought to be due to focal fibrofatty infiltration. Areas of fibrosis result in wall thinning with focally reduced contraction and aneurysm formation. These are thought to precede changes in global ventricular function, and accurate identification may improve the sensitivity of diagnosis. RV angiography was traditionally used to evaluate this, but currently MRI has replaced RV angiography due to its noninvasive nature.

Regional functional abnormalities of the RV described in ARVD include focal hypokinesis (wall thickening of $<40 \%$ ), akinesis (systolic wall thickening of $<10 \%$ ), dyskinesis (myocardial segment, which moves outward in systole), and aneurysms (segments with persistent bulging in diastole and dyskinetic in systole). Studies have consistently reported high incidence of regional dysfunction in ARVD (16-25). One study, which compared MRI to angiography, showed $86 \%$ correlation between the two modalities (17). The areas of dysfunction corresponded to the areas of signal abnormality observed on black-blood MRI. In patients presenting with arrhythmias of RV origin, the finding of isolated regional functional abnormalities limited to the RV may not be specific for the diagnosis of ARVD. But the presence of signal abnormality associated with abnormal wall motion is more suggestive of ARVD than either of them alone. In our series, $67 \%$ of the patients had regional contraction abnormalities, which correlated to the area of adipose replacement on MRI (Fig. 9A and 9B). Of these patients, 50\% had aneurysms localized to the region of adipose replacement. But overall, aneurysm formation was only observed in $25 \%$ of the patients with a final diagnosis of ARVD. 


\section{Diastolic Dysfunction}

Few investigators have used MRI to assess diastolic function in ARVD. Auffermann et al (20) were the first to use time-volume curves obtained from cine GRE MRI to assess diastolic function in biopsy-proven ARVD patients. Comparison with control subjects showed that patients with ARVD who had arrhythmias induced during electrophysiologic testing had a significant delay in diastolic relaxation of the RV. The same patients also had increased RV volumes and reduced function, so that diastolic relaxation may not provide additional diagnostic information. More recently, Kayser et al (35) evaluated diastolic function in 14 patients with ARVD with preserved systolic function using MR velocity map-
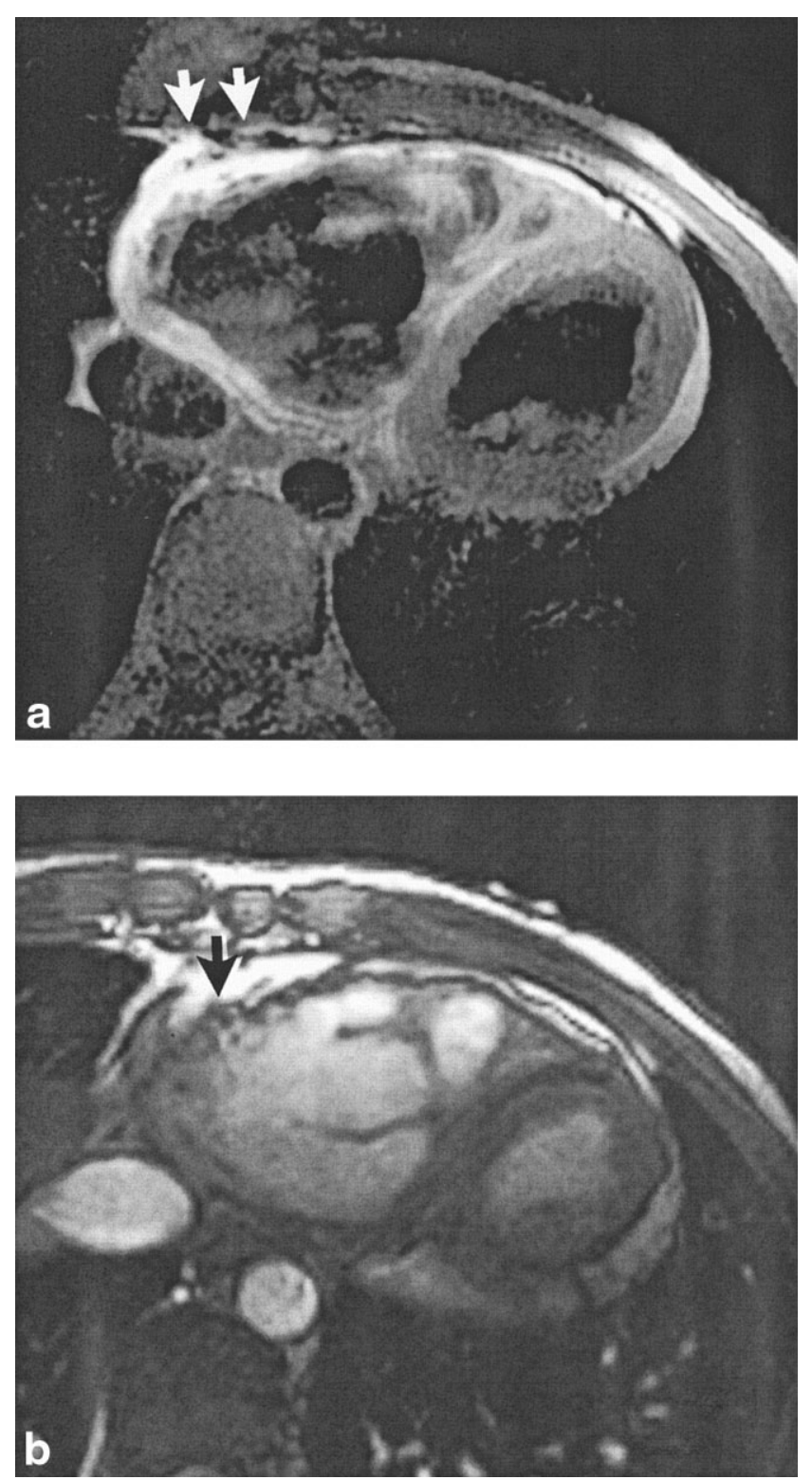

Figure 9. A: Axial black-blood image from a patient with RV dysplasia showing intramyocardial hyperintense signals (fat signal) in the anterior wall (arrows). B: Bright-blood systolic image at the same level demonstrates an aneurysmal segment (arrow) in the region of the signal abnormality. ping of transtricuspid flow. Compared to controls, tricuspid flow patterns in ARVD patients showed a significant decrease in peak filling rate and in the slope of the descending part of the early filling phase. The ratio of peak early filling rate to peak atrial contraction and ratio of integrated early filling to integrated atrial contraction (i.e., volume) were significantly lower in patients than in healthy volunteers. These data are consistent with studies using echocardiography, suggesting that diastolic abnormalities may precede systolic dysfunction and may have a role in early diagnosis.

\section{Role of MRI in Diagnosis of ARVD}

The diagnostic role of MRI in ARVD remains somewhat controversial. The ability of MRI to characterize fat in the RV free wall has been brought to question. The sensitivity and specificity of intramyocardial fat on MRI in ARVD remain to be answered. In our experience, this finding alone is neither sensitive nor specific for the diagnosis. Our experience with MRI of autopsy hearts led us to conclude that the achievable spatial resolution in current state-of-the-art clinical protocols substantially limits the capability to detect subtle RV intramyocardial fatty changes. Isolated areas of fat replacement are not specific to ARVD and have been reported in elderly patients, patients receiving long-term steroids, and in other cardiomyopathies $(36,37)$. Discrete areas of fat substitutions have also been reported in idiopathic ventricular tachycardia, which is an important differential diagnosis for ARVD (38-40).

Recently, the reliability of interpreting intramyocardial high $\mathrm{Tl}$ signals has been brought to question. Bluemke et al (41) reported poor interreader reproducibility for detection of intramyocardial fat signal on conventional SE MR images performed on patients evaluated for ARVD. The study raises an important issue in defining the role of MRI in ARVD using conventional (i.e., noncardiovascular) MR scanners. The poor agreement for identifying intramyocardial fat is not entirely surprising. The normal presence of epicardial and pericardial fat makes identification of true intramyocardial fat difficult. Some areas such as the subtricuspid region are not easily distinguished from the atrioventricular sulcus, which is rich in fat (42). The RV free wall is only 4-5 $\mathrm{mm}$ thick, and the spatial resolution is often unsatisfactory to reliably comment on wall thickness, let alone fat infiltration (43). To differentiate fatty infiltration from the normal epicardial fat requires considerable expertise, and the diagnostic sensitivity and specificity of detecting fat on MRI still need to be defined. Until these issues are resolved, the presence of intramyocardial fat on MRI should not be considered synonymous with ARVD and the diagnosis should not be made in the absence of other clinical criteria.

Since the disease is so rare, most imaging centers have little or no experience with diagnosis of ARVD. Technical problems in imaging patients with arrhythmias, lack of a standardized protocol for ARVD, and lack of experience by imaging physicians suggest that MRI should be only one part of a comprehensive evaluation for these patients. According to the Task Force 
criteria (5), MRI provides information related to RV size, global and regional function, and aneurysm formation. Even using conventional SE imaging, the morphologic features appear to distinguish ARVD patients from normal individuals (41).

MRI, on the other hand, can identify patients requiring invasive testing. A completely normal MR study in a patient with no abnormalities on electrocardiography or echocardiography is reassuring, and such patients may not need invasive testing (angiography/biopsy) in the absence of other clinical criteria. If signal abnormalities and wall motion abnormalities coexist, invasive testing should be undertaken to confirm the findings. Minor structural abnormalities, i.e., signal abnormalities in the absence of wall motion changes, present a challenge as further evaluation of such patients is unclear. Adherence to Task Force criteria is recommended, and these minor criteria may not necessitate invasive testing. Further, it must be recognized that ARVD Task Force criteria do not currently recognize fat signal on MR (or CT) as a diagnostic criterion for the disease.

\section{Future Directions}

In spite of the current problems faced by MRI, it is undeniable that MR certainly has enormous potential in ARVD. If the significant strides that have been made in MR technology over the last 10 years continue, each of the above-mentioned problems will be systematically addressed and solutions will hopefully emerge.

A current limitation for black-blood imaging as mentioned above is poor spatial resolution. Increases in spatial resolution results in longer and prohibitive breath-hold duration, which is often counterproductive. One approach would be the use of navigator echogated techniques, which allow free breathing. This technique was initially described by Ehman and Felmlee (44) in 1989 and is currently used in coronary imaging. Navigator echoes are positioned over the right diaphragm and imaging is triggered for a 4- to 5-mm window. In conjunction with three-dimensional blackblood techniques, this approach could improve spatial resolution for detection of intramyocardial fat. Limitations to this technique include increased scanning times (by a factor of two), increased edge blurring, and susceptibility to motion artifact due to arrhythmias and in patients with irregular breathing patterns.

Another aspect of ARVD evaluation that needs improvement is regional function assessment. Visual analysis is often inadequate due to the complex contraction pattern of the RV. Myocardial tissue tagging provides accurate and quantitative data regarding regional function and may potentially improve the sensitivity of MRI to detect regional dysfunction in ARVD. Fayad et al (45) have previously described this approach in the RV. However, tissue tagging is not easily applied due to the very thin RV free wall and resultant poor signal to noise ratio.

Currently, two large clinical trials in ARVD patients are under way. The European ARVD registry (46) attempts to prospectively validate criteria for clinical diagnosis of ARVD/cardiomyopathy (ARVD/C), evaluate the accuracy of clinical diagnosis, and assess the nat- ural course of the disease. The Multidisciplinary Study of Right Ventricular Dysplasia (U.S. ARVD study) (47), which aims to prospectively enroll 100 patients with ARVD and 500 first-degree relatives, attempts to develop quantitative methods to assess RV function to enhance the specificity and sensitivity of the diagnosis of ARVD/C. These studies may define the diagnostic role of MRI in ARVD as well as in family members.

\section{CT OF ARVD}

CT imaging utilizing $x$-ray technology was developed in the early 1970s, and ever since has been used extensively for its ability to provide cross-sectional images of the body (48). There has been a continuous and accelerated development of CT technology, particularly in the last 10 years, focused toward cardiac imaging. The latest-generation CT scanners, namely, electron beam CT (EBCT) and multidetector CT (MDCT), provide excellent spatial resolution and allow accurate high-resolution assessment of morphological detail of both cardiac chambers (49-51). The use of a nonionic contrast agent provides excellent contrast resolution with clear delineation of the ventricular endocardium. Multiple cardiac phases can be extracted, with animated movies of the beating heart made available for visual assessment of global and regional function (49). Quantitative determinations of ventricular mass, right and left ventricular volumes, and global ventricular function can be performed in a variety of cardiac pathologic states (50). The widespread availability of MDCT scanners and less dependence on technical factors have made CT imaging popular compared to MRI in cardiovascular evaluation. In addition, CT is fast, easy to perform, and has more reliable image quality. Although images are acquired only in the axial plane, the acquisition of a three-dimensional data set allows reformatting in any desirable plane. For the above reasons, CT is a clinically valuable, noninvasive tool for assessment of myocardial pathology.

Similar to MRI, CT imaging also has the capability to provide tissue characterization of the myocardium. The ability to depict fatty tissue along with cardiac morphology makes CT imaging an option for evaluation of ARVD. Dery et al (52) were the first to demonstrate a dilated hypokinetic RV with a markedly thin anterior wall and normal left-sided chambers using CT in an elderly patient with ARVD who presented with RV failure. However, the ability of conventional CT to detect intramyocardial fat in ARVD was first reported by Villa et al (53) in a series of seven patients with ARVD, and subsequently Sotozono et al (54) provided biopsy confirmation of CT findings. They also demonstrated the ability of CT imaging to provide excellent anatomic details of the RV and left ventricle in a patient with advanced ARVD. Since that time, there have been only a few investigators who have used CT to image ARVD, utilizing EBCT and MDCT.

\section{CT Imaging Findings in ARVD}

There has been some speculation that CT might be superior to MRI in ARVD due to factors inherent to the 


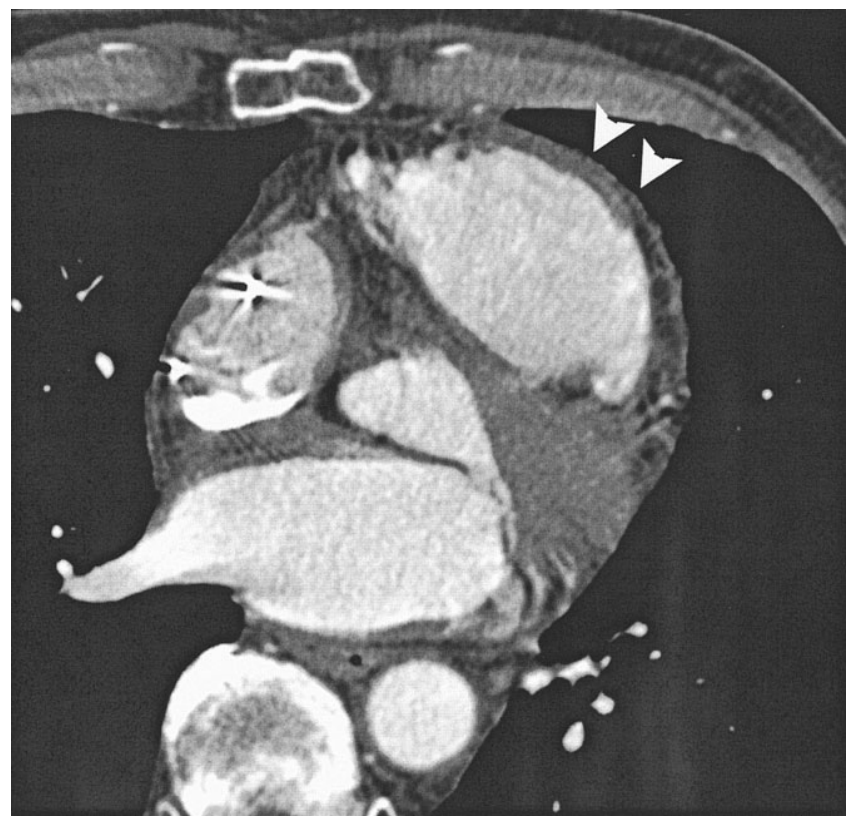

Figure 10. Contrast-enhanced, MDCT image in the axial plane showing an enlarged outflow tract (arrowheads) in a patient with RV dysplasia.

disease process. The arrhythmic nature of the disease often leads to image degradation on MR images, and ARVD patients receive defibrillator hardware, which presently precludes MRI. Hamada et al (55) imaged four ARVD patients with RV arrhythmias who had abnormalities on electrocardiography and angiography using EBCT. With contrast-enhanced volume mode scanning, they were able to demonstrate morphologic abnormalities in ARVD: 1) abundant epicardial fat, 2) low attenuation trabeculations, 3) scalloping of RV free wall, and 4) intramyocardial fat deposits. Quantification of ventricular volumes was performed on cine mode scanning, which showed regional dysfunction and depressed global RV function, respectively. Tada et al (56) added 10 more ARVD patients to the above series and compared EBCT findings to 16 age-matched non-ARVD patients with RV dilation/dysfunction and 13 control subjects. Intramyocardial fat was defined based on tissue attenuation values. The attenuation value for epicardial adipose tissue is around $-65 \pm 10$ Hounsfield units (HU), and 5 to $-17 \mathrm{HU}$ for intramyocardial fat, which is far less than that of myocardium. Using the above values, none of the control subjects and no patient without ARVD showed any evidence of intramyocardial fat or any other qualitative features of ARVD, as described by Tada et al (56). The frequencies of abundant epicardial fat, low-attenuation trabeculae, scalloping, and intramyocardial fat in this study were $86 \%$, $71 \%, 79 \%$, and 50\%, respectively. An important finding of this study was that the abnormal area on EBCT corresponded to the areas of abnormality on electroanatomic mapping and was frequently larger than the electroanatomic maps.

Kimura et al (57) studied 32 ARVD patients using contrast-enhanced, nongated, single-row detector helical CT. Similar to the findings of EBCT, they found intramyocardial fat, RV enlargement, hypertrophied trabeculations, and abundant epicardial fat in patients with ARVD. This study also provided radiologic and pathologic correlation in one autopsy heart with ARVD, illustrating the applicability of widely available helical CT in ARVD evaluation. There have been no reports on the use of MDCT in ARVD yet in the literature. Our group studied 13 prospectively diagnosed ARVD patients with MDCT (four detectors), and the results were recently presented in abstract form (58). We found good correlation between RV inflow diameter (measured $1 \mathrm{~cm}$ below tricuspid valve) and RV end-diastolic volume, suggesting that this might be a crude surrogate to RV enlargement. Enlargement of the RVOT was also noted in the majority of patients (61\%), and this too correlated with increased RV volumes (Fig. 10). Left ventricular involvement with focal wall thinning and fat infiltration of the inferior wall was observed in only one of the 13 patients (Fig. 11).

\section{Current Role of CT in ARVD}

The majority of patients who are diagnosed with ARVD receive implantable defibrillators for prevention of sudden death. Defibrillator artifacts do degrade image quality on MRI, and quantification of RV function may be compromised. In this cohort CT remains the only imaging technique to assess RV structure and function for serial morphologic evaluation. CT is also useful in the occasional patient who has frequent premature beats resulting in arrhythmic artifacts on MRI and in patients who are claustrophobic. An additional use of CT imaging is to assess the mediastinum for presence of lymphadenopathy, a marker of sarcoidosis, which occasionally mimics ARVD (59).

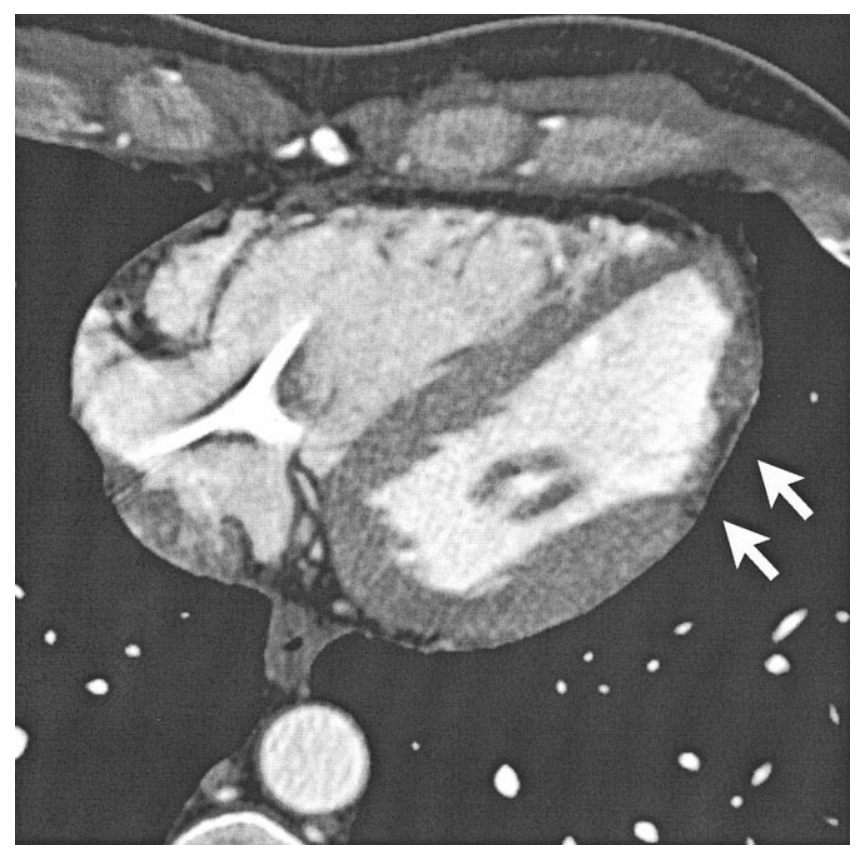

Figure 11. Contrast-enhanced, MDCT image in the axial plane showing focal fat infiltration of the left ventricle with wall thinning (arrows) in a patient with RV dysplasia. 
Most centers currently rely on MRI instead of CT imaging for evaluating patients with suspected ARVD, mainly because the former is devoid of radiation. MDCT radiation can be quite high, exceeding conventional angiography by a factor of two when performing retrospective gating (60). Thus, MDCT may not be optional for screening of ARVD in first-degree relatives. This would be of particular importance especially in young females in whom breast radiation should be considered. Current temporal resolution of CT is of the order of 105 msec (at a heart rate of 81 beats/minute), but is still suboptimal compared to MRI (61). Despite the above limitations, CT does provide certain advantages over MRI in terms of consistency in image quality, scan time, operator dependency, etc. With increase in familiarity of radiologists with the use of helical CT for ARVD and with the advances in both the temporal and spatial resolution, CT imaging may play an important role both in the diagnosis and in the follow-up of patients with ARVD.

\section{Future Directions}

Current multiple-row detector scanners can scan 40cm-volume lengths in less than 30 seconds with submillimeter near-isotropic resolution and excellent image quality. Higher spatial resolution may allow accurate and reproducible evaluation of intramyocardial fat and RV wall thickness, thereby increasing the sensitivity in ARVD diagnosis. Increasing gantry speeds will improve temporal resolution approaching that of MRI. Accurate calculation of radiation dose with customized adjustment individualized to a patient's body habitus may increase the utilization of CT in ARVD. Studies comparing MRI and MDCT in ARVD would further our knowledge of the relative utility of each of these technologies in evaluation of ARVD.

\section{ACKNOWLEDGMENTS}

This work was supported in part by National Institutes of Health Research Grant 1 UO1 HL65594-01A1 (to D. Bluemke, PI, MRI ARVD reading center).

\section{REFERENCES}

1. Marcus FI, Fontaine GH, Guiraudon G, et al. Right ventricular dysplasia: a report of 24 adult cases. Circulation 1982;65:384-398.

2. Laurent M, Descaves C, Biron Y, et al. Familial form of arrhythmogenic right ventricular dysplasia. Am Heart J 1987;113:827-829.

3. Corrado D, Thiene G, Nava A, Rossi L, Pennelli N. Sudden death in young competitive athletes: clinicopathologic correlations in 22 cases. Am J Med 1990;89:588-596.

4. Thiene G, Nava A, Corrado D, Rossi L, Penelli N. Right ventricular cardiomyopathy and sudden death in young people. N Engl J Med 1988;318:129-133.

5. McKenna WJ, Thiene G, Nava A, et al. Diagnosis of arrhythmogenic right ventricular dysplasia/cardiomyopathy. Task Force of the Working Group Myocardial and Pericardial Disease of the European Society of Cardiology and of the Scientific Council on Cardiomyopathies of the International Society and Federation of Cardiology. Br Heart J 1994;71:215-218.

6. Boxt LM. Radiology of the right ventricle. Radiol Clin North Am 1999;37:379-400.

7. Foale R, Nihoyannopoulos P, McKenna W, et al. Echocardiographic measurement of the normal adult right ventricle. Br Heart J 1986; $56: 33-44$.
8. Pettigrew RI, Oshinski JN, Chatzimavroudis G, Dixon WT. MRI techniques for cardiovascular imaging. J Magn Reson Imaging 1999;10:590-601.

9. Higgins CB, editor. Special issue: cardiovascular MRI. J Magn Reson Imaging 1999;10:589-899.

10. Araoz PA, Mulvagh SL, Tazelaar HD, Julsrud PR, Breen JF. CT and MR imaging of benign primary cardiac neoplasms with echocardiographic correlation. Radiographics 2000;20:1303-1319.

11. Becker CR, Ohnesorge BM, Schoepf UJ, Reiser MF. Current development of cardiac imaging with multidetector-row CT. Eur J Radiol 2000;36:97-103.

12. Casolo GC, Poggesi L, Boddi M, Fazi A, Bartolozzi C, Lizzadro G, Dabizzi RP. ECG-gated magnetic resonance imaging in right ventricular dysplasia. Am Heart J 1987;113:1245-1248.

13. Klersy C, Raisaro A, Salerno JA, Montemartini C, Campani R. Arrhythmogenic right and left ventricular disease: evaluation by computed tomography and nuclear magnetic resonance imaging. Eur Heart J 1989;10(Suppl D):33-36.

14. Bloomer TN, Plein S, Radjenovic A, et al. Cine MRI using steady state free precession in the radial long axis orientation is a fast accurate method for obtaining volumetric data of the left ventricle. J Magn Reson Imaging 2001;14:685-692.

15. Simonetti OP, Finn JP, White RD, Laub G, Henry DA. "Black blood" T2-weighted inversion-recovery MR imaging of the heart. Radiology 1996; 199:49-57.

16. Tandri $\mathrm{H}$, Calkins $\mathrm{H}$, Nasir $\mathrm{K}$, et al. MR imaging findings in patients meeting task force criteria for arrhythmogenic right ventricular dysplasia. J Cardiovasc Electrophysiol 2003;14:476-483.

17. Midiri M, Finazzo M, Brancato M, Hoffmann E, Indovina G, Maria MD, Lagalla R. Arrhythmogenic right ventricular dysplasia: MR features. Eur Radiol 1997;7:307-312.

18. Pennell D, Casolo G. Right ventricular arrhythmia: emergence of magnetic resonance imaging as an investigative tool. Eur Heart J 1997; 18:1843-1845.

19. van der Wall EE, Kayser HW, Bootsma MM, de Roos A, Schalij MJ. Arrhythmogenic right ventricular dysplasia: MR imaging findings. Herz 2000;4:356-364.

20. Auffermann W, Wichter T, Breithardt G, Joachimsen K, Peters PE. Arrhythmogenic right ventricular disease: MR imaging vs. angiography. AJR Am J Roentgenol 1993;161:549-555.

21. Wichter T. Magnetic resonance imaging and ${ }^{123}$ I-MIBG scintigraphy in arrhythmogenic right ventricular cardiomyopathy/dysplasia. In: First International Symposium on Arrhythmogenic Right Ventricular Cardiomyopathy/Dysplasia, Paris, June 16-18, 1996.

22. Ricci C, Longo R, Pagnan L, et al. Magnetic resonance imaging in right ventricular dysplasia. Am J Cardiol 1992;70:1589-1595.

23. Blake LM, Scheinman MM, Higgins CB. MR features of arrhythmogenic right ventricular dysplasia. AJR Am J Roentgenol 1994;162: 809-812.

24. Molinari G, Sardanelli F, Gaita F, et al. Right ventricular dysplasia as a generalized cardiomyopathy? Findings on magnetic resonance imaging. Eur Heart J 1995;16:1619-1624.

25. Menghetti L, Basso C, Nava A, Angelini A, Thiene G. Spin-echo nuclear magnetic resonance for tissue characterisation in arrhythmogenic right ventricular cardiomyopathy. Heart 1996;76:467-470.

26. van der Wall EE, Kayser HW, Bootsma MM, de Roos A, Schalij MJ. Arrhythmogenic right ventricular dysplasia: MRI findings. Herz 2000;25:356-364.

27. Arai AE, Epstein $\mathrm{FH}$, Bove $\mathrm{KE}$, Wolff SD. Visualization of aortic valve leaflets using black blood MRI. J Magn Reson Imaging 1999; 10:771-777.

28. Fayad ZA, Fuster V, Fallon JT, et al. Noninvasive in vivo human coronary artery lumen and wall imaging using black-blood magnetic resonance imaging. Circulation 2000;102:506-510.

29. Stuber M, Botnar RM, Kissinger KV, Manning WJ. Free-breathing black-blood coronary MR angiography: initial results. Radiology 2001;219:278-283.

30. Burke AP, Farb A, Tashko G, Virmani R. Arrhythmogenic right ventricular cardiomyopathy and fatty replacement of the right ventricular myocardium: are they different diseases? Circulation 1998;97:1571-1580.

31. Sakuma H, Fujita N, Foo TK, et al. Evaluation of left ventricular volume and mass with breath-hold cine MR imaging. Radiology 1993;188:377-380.

32. Bloomgarden DC, Fayad ZA, Ferrari VA, et al. Global cardiac function using fast breath-hold MRI: validation of new acquisition and analysis techniques. Magn Reson Med 1997;37:683-692. 
33. Pereles FS, Kapoor V, Carr JC, et al. Usefulness of segmented trueFISP cardiac pulse sequence in evaluation of congenital and acquired adult cardiac abnormalities. AJR Am J Roentgenol 2001; 177:1155-1160.

34. Candinas R, Duru F. Unusual clinical presentation of a patient with an extreme form of right ventricular dysplasia. Circulation $2001 ; 104: 848-849$.

35. Kayser HW, de Roos A, Schalij MJ, Bootsma M, Wellens HJ, Van der Wall EE. Usefulness of magnetic resonance imaging in diagnosis of arrhythmogenic right ventricular dysplasia and agreement with electrocardiographic criteria. Am J Cardiol 2003;91:365-367.

36. Vikhert AM, Tsiplenkova VG, Cherpachenko NM. Alcoholic cardiomyopathy and sudden cardiac death. J Am Coll Cardiol 1986; 8(Suppl A):3A-11A.

37. Hasumi M, Sekiguchi M, Hiroe M, Kasanuki H, Hirosawa K. Endomyocardial biopsy approach to patients with ventricular tachycardia with special reference to arrhythmogenic right ventricular dysplasia. Jpn Circ J 1987;51:242-249.

38. White RD, Trohman RG, Flamm SD, et al. Right ventricular arrhythmia in the absence of arrhythmogenic dysplasia: MR imaging of myocardial abnormalities. Radiology 1998;207:743-751.

39. Proclemer A, Basadonna PT, Slavich GA, Miani D, Fresco C, Fioretti PM. Cardiac magnetic resonance imaging findings in patients with right ventricular outflow tract premature contractions. Eur Heart J 1997;18:2002-2010.

40. Globits S, Kreiner G, Frank H, Heinz G, Klaar U, Frey B, Gossinger H. Significance of morphological abnormalities detected by MRI in patients undergoing successful ablation of right ventricular outflow tract tachycardia. Circulation 1997;96:2633-2640.

41. Bluemke D, Krupinski EA, Ovitt T, et al. MR imaging in arrhythmogenic right ventricular cardiomyopathy: morphologic findings and interobserver reliability. Cardiology 2003;99:153-162.

42. Pennell D. Cardiovascular magnetic resonance. Heart 2001;85: 581-589.

43. Corrado D, Basso C, Thiene G. Arrhythmogenic right ventricular cardiomyopathy: diagnosis, prognosis, and treatment. Heart 2000; 83:588-595.

44. Ehman RL, Felmlee JP. Adaptive technique for high-definition MR imaging of moving structures. Radiology 1989;173:255-263.

45. Fayad ZA, Ferrari VA, Kraitchman DL, et al. Right ventricular regional function using MR tagging: normal versus chronic pulmonary hypertension. Magn Reson Med 1996;35:771-780.

46. Corrado C, Fontaine G, Marcus FI, et al., for the Study Group on Arrhythmogenic Right Ventricular Dysplasia/Cardiomyopathy. Arrhythmogenic right ventricular dysplasia/cardiomyopathy need for an international registry. Circulation 2000;101:E101-E106.
47. Marcus FI, Towbin JA, Zareba W, et al., for the ARVD/C Investigators. Arrhythmogenic right ventricular dysplasia/cardiomyopathy (ARVD/C). A multidisciplinary study: design and protocol. Circulation 2003;107:2975-2978.

48. Hounsfield GN. Computerized transverse axial scanning (tomography). 1. Description of system. Br J Radiol 1973;46:1016-1022.

49. Berry E, Kelly S, Hutton J, Harris KM, et al. A systematic literature review of spiral and electron beam tomography: with particular reference to clinical application in hepatic lesions, pulmonary embolus and coronary artery disease. Health Technol Assess 1999;3: 1-118.

50. Stanford W, Galvin JR, Weiss RM, et al. Ultrafast computed tomography in cardiac imaging: a review. In: Seminars in Ultrasound, CT and MR, 1991. p 45-60.

51. Becker CR, Ohnesorge BM, Schoepf UJ, Reiser MF. Current development of cardiac imaging with multidetector-row CT. Eur J Radiol 2000;36:97-103.

52. Dery R, Lipton MJ, Garrett JS, Abbott J, Higgins CB, Schienman MM. Cine-computed tomography of arrhythmogenic right ventricular dysplasia. J Comput Assist Tomogr 1986;10:120-123.

53. Villa A, Di Guglielmo L, Salerno J, Klercy C, Kluzer A, Codega S. Arrhythmogenic dysplasia of the right ventricle. Evaluation of 7 cases using computerized tomography. Radiol Med (Torino) 1988; 75:28-35.

54. Sotozono K, Imahara S, Masuda H, et al. Detection of fatty tissue in the myocardium by using computerized tomography in a patient with arrhythmogenic right ventricular dysplasia. Heart Vessels Suppl 1990;5:59-61.

55. Hamada S, Takamiya M, Ohe T, Ueda H. Arrhythmogenic right ventricular dysplasia: evaluation with electron-beam CT. Radiology 1993; 187:723-727.

56. Tada H, Shimizu W, Ohe T, et al. Usefulness of electron-beam computed tomography in arrhythmogenic right ventricular dysplasia. Circulation 1996;94:437-444.

57. Kimura F, Sakai F, Sakomura Y, et al. Helical CT features of arrhythmogenic right ventricular cardiomyopathy. Radiographics 2002;22:1111-1124.

58. Bomma C, Tandri H, Nasir K, et al. Role of helical CT in qualitative and quantitative evaluation of arrhythmogenic right ventricular dysplasia. Pacing Clin Electrophysiol 2003;26(Suppl 1):965.

59. Shiraishi J, Tatsumi T, Shimoo K, et al. Cardiac sarcoidosis mimicking right ventricular dysplasia. Circ J 2003;67:169-171.

60. Hunold P, Vogt FM, Schmermund A, et al. Radiation exposure during cardiac CT: effective doses at multi-detector row CT and electron-beam CT. Radiology 2003;226:145-152.

61. Flohr TG, Schoepf UJ, Kuettner A, et al. Advances in cardiac imaging with 16-section CT systems. Acad Radiol 2003;10:386-401. 